\title{
Monitoring Bone Marrow Stem Cells with a Reporter Gene System in Experimental Middle Cerebral Artery Occlusion Rat Models
}

\author{
Tao $\mathrm{Wu}^{1-3}$, Juntao Lang ${ }^{1,2}$, Xun Sun ${ }^{1,2}$, Binqing Zhang ${ }^{1,2}$, Yu Liu ${ }^{1,2}$, and Rui An ${ }^{1,2}$ \\ ${ }^{1}$ Department of Nuclear Medicine, Union Hospital, Huazhong University of Science and Technology, Wuhan, China; ${ }^{2}$ Molecular \\ Imaging Key Laboratory of Hubei, Wuhan, China; and ${ }^{3}$ Department of Nuclear Medicine, The Second People's Hospital of Wuhu, \\ Wuhu, China
}

This study was designed to investigate the feasibility of imaging bone marrow stem cells (BMSCs) in experimental middle cerebral artery occlusion (MCAO) rat models with a reporter gene-probe system, HSV1-tk-131I-2' -fluoro-2' -deoxy-1- $\beta$-D-arabinofuranosyl5 -iodouracil ( $\left.{ }^{131} \mathrm{I}-\mathrm{FIAU}\right)$, and to choose the best strategies for stem cell injection, image acquisition, and imaging in vivo. Methods: A recombinant adenovirus (Ad5-TIBE) carrying the herpes simplex virus type 1 thymidine kinase (TK) reporter gene (HSV1-tk) linked via the internal ribosome entry site to the brain-derived neurotrophic factor therapeutic gene was prepared. After transfection with Ad5-TIBE, BMSCs were introduced into MCAO rat models via local injection into the brain or via injection into the lateral ventricle, carotid artery, and tail vein. Normal rats were used as controls. Twenty-four hours after ${ }^{131}$ I-FIAU injection, rats were sacrificed for biodistribution analysis. The expression of the TK gene was evaluated by real-time quantitative polymerase chain reaction and Western blot analysis. Autoradiography was used for ex vivo imaging. SPECT images were obtained in MCAO rat models. Results: The percentage injected dose per gram (\%ID/g) in infarcted brain tissue in rats receiving the injection into the brain was $0.124 \pm 0.013$; this value was significantly higher than those in rats receiving the injection into the ventricle $(0.052 \pm 0.004)$, carotid artery $(0.061 \pm 0.002)$, and tail vein $(0.059 \pm 0.005)$ as well as normal rats $(0.005 \pm 0.001)$. No differences were seen in the other cell transplantation groups. The \%ID/g in infarcted brain tissue was higher than that in the contralateral brain tissue in all experimental rats but not in normal rats. The expression of the TK gene in rats receiving a local injection into the brain was superior to that in all of the other groups. TK messenger RNA and protein expression showed a positive correlation with the $\% I D / g$ in brain tissue. Greater radioactivity at the injection site than in the surrounding and contralateral brain tissues in all experimental rats was indicated through autoradiography. The ratio of counts in bilateral brain tissues reached its peak (6.63) $24 \mathrm{~h}$ after ${ }^{131} \mathrm{I}$-FIAU injection. SPECT images showed that radioactivity accumulation in the brain was low but increased gradually over time. Conclusion: The HSV1-tk- ${ }^{131}$ I-FIAU reporter gene-probe system may be used to monitor BMSC activity in experimental MCAO rat models. Local injection of stem cells

Received May 25, 2012; revision accepted Dec. 11, 2012.

For correspondence or reprints contact: Rui An, 1277 Jiefang Rd., Wuhan, Hubei Province, China.

E-mail: anruiwh@163.com

Published online Mar. 27, 2013.

COPYRIGHT @ 2013 by the Society of Nuclear Medicine and Molecular Imaging, Inc. may provide an optimal means for cell transplantation, and imaging with ${ }^{131} \mathrm{I}$-FIAU $24 \mathrm{~h}$ after injection provides peak target-tonontarget count ratios.

Key Words: reporter gene; middle cerebral artery occlusion; biodistribution; autoradiography; SPECT

J Nucl Med 2013; 54:984-989

DOI: 10.2967/jnumed.112.109280

$\mathbf{R}$ ecently, the efficacy of bone marrow stem cells (BMSCs) as a transport vector for gene therapy in various diseases has become a focus of research. These cells are derived from bone marrow and can differentiate into various cell types, including neurons and glial cells $(1,2)$.

Previous studies showed that transplantation of BMSCs into experimental cerebral ischemia rat models via intracerebral, intravenous, or intraarterial routes can reduce infarct size and promote functional improvements (3-5). Furthermore, other reports showed that transplantation of marrow stem cells modified with the brain-derived neurotrophic factor (BDNF) gene is more effective than transplantation of nonmodified marrow stem cells in promoting functional improvements $(6,7)$. However, analysis of the functional improvements in brain tissue in these studies was mainly performed in vitro after animals were sacrificed. Recently, several techniques, including intravital multiphoton microscopy, bioluminescence, PET, and MR imaging, were used for noninvasive cellular imaging in vivo $(8-10)$. In addition, Walczak et al. (11) used laser Doppler flow signal measurement and MR imaging for noninvasive monitoring of the cells. Noninvasive tracer techniques are becoming the focus of many researches.

Over the past few years, reporter gene systems have been increasingly used for monitoring cell or gene therapy in various diseases. Now, HSV1-tk- ${ }^{131} \mathrm{I}-2$ '-fluoro-2'-deoxy-1$\beta$-D-arabinofuranosyl-5-iodouracil ( ${ }^{131}$ I-FIAU) is one of the most mature reporter gene systems $(12,13)$. Unfortunately, a previous study demonstrated that radiolabeled FIAU could not penetrate the blood-brain barrier (14), and this factor 
greatly limited its application in cerebrovascular diseases. Nevertheless, experimentally induced brain infarcts can damage the blood-brain barrier, supplying the necessary condition for the use of this reporter gene system. Therefore, the aims of this study were to investigate the feasibility of imaging HSV1-tk with ${ }^{131}$ I-FIAU, autoradiography (ARG), and SPECT and to investigate optimal conditions for further imaging in vivo. In addition, this study is the first to apply different methods of stem cell transplantation into middle cerebral artery occlusion (MCAO) rat models and to evaluate the feasibility of nuclear reporter gene imaging for monitoring stem cells and therapeutic genes.

\section{MATERIALS AND METHODS}

\section{Adenoviral Vector}

An adenoviral vector (Ad5-TIBE) carrying the HSV1-tk, BDNF, and enhanced green fluorescent protein (EGFP) genes under the control of the cytomegalovirus promoter and Ad5-EGFP were constructed by Ming Hong Biology Co. Ltd. The total yields of Ad5TIBE and Ad5-EGFP were $2 \times 10^{10}$ and $3.6 \times 10^{10}$ plaque-forming units $/ \mathrm{mL}$, respectively.

\section{Experimental MCAO Models}

This study was approved by the Institutional Animal Care and Use Committee at Huazhong University of Science and Technology. Adult male Sprague-Dawley rats weighing 250-300 g (supplied by the Experimental Animal Center of Tongji Medical Collage, Huazhong University of Science and Technology) were used to prepare the experimental MCAO models by a modification of the method of Kuge et al. (15). Animals were initially anesthetized intraperitoneally with $10 \%$ chloral hydrate at $0.3 \mathrm{~mL} / 100 \mathrm{~g}$ of weight. Rat body temperature was maintained at $37^{\circ} \mathrm{C}$ with a heat lamp throughout the surgical procedure. The left common carotid artery, external carotid artery, and internal carotid artery were exposed under anesthesia. A 4-0 monofilament nylon suture with a tip rounded by flame heating was inserted from the left common carotid artery into the internal carotid artery and then advanced approximately $18 \mathrm{~mm}$ intracranially from the common carotid artery bifurcation. Sixty minutes after MCAO, rats were reanesthetized, and reperfusion was performed by withdrawal of the suture. All animals underwent behavioral testing $24 \mathrm{~h}$ after MCAO. A previously described (16) 5-point system was used to assess the severity of injury. Rats with scores ranging from 1 (failure to extend forepaw fully) to 3 (falling) points were used.

\section{Cell Preparation and Transplantation}

BMSCs were isolated and cultured from the bone marrow of Sprague-Dawley rats. Cells in the exponential growth phase were seeded at a density of $5 \times 10^{5} /$ well of 6 -well culture plates. After reaching fusion, the cells were infected with Ad5-TIBE at a multiplicity of infection of 150 plaque-forming units per cell. The cells were exposed to the viral particles at $37^{\circ} \mathrm{C}$ for $2 \mathrm{~h}$, the medium was removed, and the cells were washed once with phosphate-buffered saline (PBS) and recultured with normal medium for $36 \mathrm{~h}$. Next, the cells were harvested for transplantation.

Previous studies showed that cell transplantation in the acute phase after MCAO can lead to more obvious functional improvements $(17,18)$. Therefore, cell transplantation was performed $1 \mathrm{~d}$ after MCAO in this study. After the cells were prepared, a suspension containing $2 \times 10^{6}$ cells was collected and centrifuged for
$5 \mathrm{~min}$ at 1,000 rpm, and then the cells were suspended in $15 \mu \mathrm{L}$ of PBS. A $25-\mu \mathrm{L}$ microsyringe affixed to the stereotactic instrument was used to pump the suspension. The stereotactic coordinates for cell local injection were $1 \mathrm{~mm}$ before the bregma, $3 \mathrm{~mm}$ lateral to the midline, and $5 \mathrm{~mm}$ beneath the skull surface; the coordinates for ventricular injection were $1 \mathrm{~mm}$ after the bregma, $1.5 \mathrm{~mm}$ lateral to the midline, and $3.5 \mathrm{~mm}$ beneath the skull surface. A suspension of $2 \times 10^{6}$ cells in $15 \mu \mathrm{L}$ of PBS was injected into each rat that had undergone MCAO over a 5-min period on the stereotactic apparatus (Ruiwode Life Technology Co., Ltd.). However, for carotid artery and tail vein injections, a $0.5-\mathrm{mL}$ cell suspension was prepared. All animals received penicillin $\left(2 \times 10^{5} \mathrm{U}\right.$ daily $)$ intramuscularly as prophylaxis, and no immunosuppressant was used in any animal.

\section{Radioiodination of 2'-Fluoro-2' -Deoxy-1- $\beta$-D- Arabinofuranosyl-Uracil}

2'-Fluoro-2'-deoxy-1- $\beta$-D-arabinofuranosyl-uracil (Moravek Biochemicals) was labeled according to the method described by Lan et al. (19). The product was purified with methanol on a reverse-phase Sep-Pak C-18 column (Waters). The final product ( ${ }^{131}$ I-FIAU) was collected to determine biochemical purity with thin-layer chromatography in an acetoacetate-acetone-water $(12: 8: 1, \mathrm{v} / \mathrm{v})$ solution. The resulting product was incubated in PBS and human serum at $37^{\circ} \mathrm{C}$ for 12 and $24 \mathrm{~h}$ to verify stability.

\section{Biodistribution}

For biodistribution experiments, cells were transplanted into MCAO models via local injection $(n=5)$, ventricular injection $(n=5)$, carotid artery injection $(n=5)$, and tail vein injection $(n=5)$. Normal rats were used as a control group $(n=4)$. The animals were sacrificed $24 \mathrm{~h}$ after injection of $1.11 \mathrm{MBq}$ of ${ }^{131}$ I-FIAU into each rat. Bilateral brain, thyroid, lung, heart, liver, stomach, pancreas, spleen, kidney, muscle, bone, blood, and small intestine tissues were extracted; their wet weights were measured, and $\gamma$-counts were determined. After correction for radioactive decay, the percentage injected dose per gram $(\% \mathrm{ID} / \mathrm{g})$ and the ratio of the $\% \mathrm{ID} / \mathrm{g}$ in infarcted brain to the $\% \mathrm{ID} / \mathrm{g}$ in blood were calculated.

\section{Real-Time Quantitative Polymerase Chain Reaction and Western Blotting}

Total RNA was extracted from bilateral brain tissues, and complementary DNA was synthesized according to the instructions in a Maxima First Strand cDNA Synthesis Kit (Thermo Scientific). The primer sequences were $5^{\prime}$-CTCACCCTCATCTTCGACCG-3' (forward) and 5'-CCTGCAGATACCGCACCGTA-3' (reverse). Amplification for 45 cycles was performed by use of a Real-time PCR Detection System (Applied Biosystems) with those primer sequences; the total reaction volume was $10 \mu \mathrm{L}$.

The brain tissue was removed immediately under anesthesia, ground completely, and centrifuged at $12,000 \mathrm{rpm}$ and $4^{\circ} \mathrm{C}$ for $5 \mathrm{~min}$. The supernatant was collected for protein quantitation by use of a bicinchoninic acid protein assay kit (Beyotime Biotechnology). Fifty micrograms of protein were loaded onto a sodium dodecyl sulfate-polyacrylamide gel for electrophoresis and electroblotted onto a polyvinylidene difluoride membrane (Invitrogen). The membrane was incubated with anti-thymidine kinase (TK) (1:500) overnight at $4^{\circ} \mathrm{C}$, and $\beta$-actin was used as a control. AlphaEaseFC software, version 4.0 (Alpha Innotech), was used to verify the level of expression of the HSV1-tk gene. Each experiment was repeated in triplicate. 


\section{ARG}

For ARG experiments, cells were transplanted into the brain via local injection. Twelve hours after cell transplantation, $1.11 \mathrm{MBq}$ of ${ }^{131}$ I-FIAU were injected via the tail vein. Rats were divided into 4 groups as follows. In groups 1 and 2, the probes ${ }^{131}$ I-FIAU (group $1, n=5$ ) and ${ }^{131} \mathrm{I}$ (group 2, $n=5$ ) were injected into MCAO rat models with BMSCs transfected with Ad5-TIBE. In group 3, ${ }^{131}$ I-FIAU $(n=5)$ was injected into MCAO rat models transfected with Ad5-EGFP-modified BMSCs. In group 4, ${ }^{131}$ IFIAU $(n=5)$ was injected into normal rats that had undergone transplantation with nontransfected BMSCs. Four rats randomly selected from each group were sacrificed at 2, 12, 24, and $48 \mathrm{~h}$ after injection. The brain tissue was removed immediately and prepared for frozen sectioning. After a series of 15- $\mu \mathrm{m}$-thick sections were obtained, ARG was performed with a Cyclone Storage Phosphor System (PerkinElmer), and the counts in the images were analyzed with PerkinElmer OptiQuant image analysis software (PerkinElmer). The ratio of counts in bilateral brain tissues was calculated.

\section{SPECT Imaging}

As with the ARG experiments, cells were transplanted into the brain via local injection, and then $1.11 \mathrm{MBq}$ of ${ }^{131}$ I-FIAU were injected. SPECT imaging was performed with rats in the prone position at 2, 8, and $24 \mathrm{~h}$ after probe injection. The matrix was 512 $\times 512$, and the zoom was 2 . Image acquisition was stopped when counts reached 120,000 .

\section{Statistical Analysis}

Data are presented as mean \pm SE and were analyzed with SPSS 11.5 software (SPSS Inc.). The statistical significance level was set at a $P$ value of less than 0.05 . For analysis of differences, we adopted the independent-samples $t$ test for 2 groups and the Levene test, the least significant difference $t$ test, and the KruskalWallis test for multiple groups.

\section{RESULTS}

\section{MCAO Models and Behavioral Testing}

Rats with 1-3 points were chosen for all experiments. There were no statistical differences among all experimental groups in all experiments.

\section{Radiolabeling and Stability}

The efficiency of radiolabeling of ${ }^{131}$ I-FIAU was $62.89 \% \pm$ $2.46 \%(n=5)$. After purification on the Sep-Pak C-18 column, the radiochemical purity of the final product $\left({ }^{131}\right.$ I-FIAU) was $98.22 \% \pm 0.34 \%(n=5)$. The radiochemical purity of ${ }^{131}$ I-FIAU remained above $90 \%$ after incubation in serum and $\mathrm{PBS}$ at $37^{\circ} \mathrm{C}$ for 12 and $24 \mathrm{~h}$, indicating that ${ }^{131}$ I-FIAU was stable in both serum and PBS.

\section{Biodistribution}

The $\% \mathrm{ID} / \mathrm{g}$ in infarcted brain tissue after injection of BMSCs was higher than that in the contralateral brain tissue in all cell injection groups in MCAO models $(t=9.00$ 15.73; $P<0.01)$ but not in the control group $(t=1.51 ; P=$ $0.182)$. The $\% \mathrm{ID} / \mathrm{g}$ in infarcted brain tissue in rats receiving the injection into the brain was $0.124 \pm 0.013$; this value was significantly higher than those in rats receiving the injection into the ventricle $(0.052 \pm 0.004)$, carotid artery
$(0.061 \pm 0.002)$, and tail vein $(0.059 \pm 0.005)$ as well as normal rats $(0.005 \pm 0.001)(P<0.05)$. No statistical differences were seen in the other cell transplantation groups $(P>0.05)$ (Fig. 1). The ratio of the \% ID/g in infarcted brain to the \% ID/g in blood was highest $(7.765 \pm$ 3.081) in rats receiving a local injection into the brain (Table 1).

\section{Expression of Reporter Gene}

The level of expression of the TK gene in infarcted brain tissue was higher than that in contralateral brain tissue in all experimental MCAO rat models $(t=26.14-122.44 ; P<$ $0.001)$. There were statistically significant differences in TK gene expression in the infarcted brain between the local injection group and groups injected through other routes $(P<0.05)$ but not among the latter groups $(P>$ 0.05). TK gene expression from the real-time quantitative polymerase chain reaction showed a positive correlation with the $\% \mathrm{ID} / \mathrm{g}$ in brain tissue $\left(R^{2}=0.971 ; P<0.001\right)$ (Fig. 2).

The ratio of TK to $\beta$-actin in infarcted brain tissue was higher than that in contralateral brain tissue in all experimental MCAO rat models $(t=33.10-117.87 ; P<0.001)$. There were statistically significant differences in the ratio of TK to $\beta$-actin in the infarcted brain between the local injection group and groups injected through other routes $(P<0.05)$ but not among the latter groups $(P>0.05)$. The ratio of TK to $\beta$-actin on Western blotting and the $\% \mathrm{ID} / \mathrm{g}$ also correlated well $\left(R^{2}=0.899 ; P=0.002\right)$ (Fig. 3).

\section{ARG}

As indicated on ARG images (Fig. 4), radioactivity accumulation at the injection site in all experimental rats was greater than that in the surrounding tissue $(P<0.05)$ and contralateral brain tissue $(P<0.001)$. There was no significant difference between bilateral brain tissues in normal rats $(P>0.05)$. The counts progressively decreased over

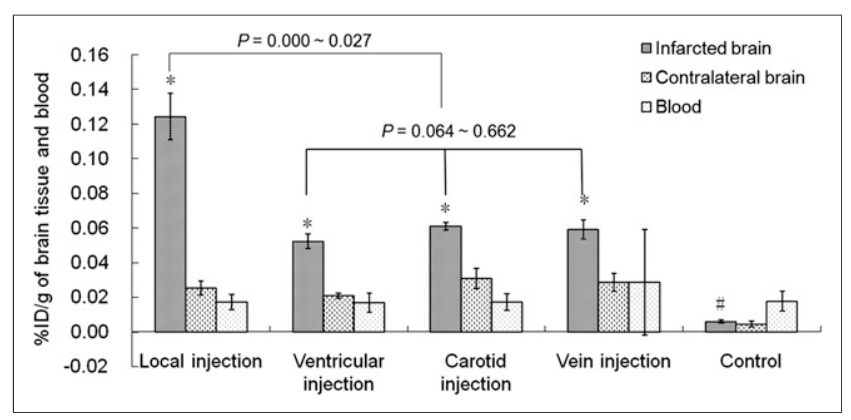

FIGURE 1. \%ID/g in bilateral brain tissues and blood $24 \mathrm{~h}$ after injection of ${ }^{131} \mathrm{I}-\mathrm{FIAU} . \% \mathrm{ID} / \mathrm{g}$ in infarcted brain tissue was higher than that in contralateral brain tissue in all cell injection groups $\left({ }^{*} P<\right.$ 0.01 ) but not in control group ( $P=0.182$ ). Furthermore, \%ID/g in infarcted brain tissue was higher in local injection group than in ventricular, carotid artery, and vein injection groups. Ratio of $\% \mathrm{ID} / \mathrm{g}$ in infarcted brain to \% ID/g in blood was highest $(7.765 \pm 3.081)$ in local injection group (Table 1). 
TABLE 1

Biodistribution of ${ }^{131}$ I-FIAU in MCAO Rat Models

\begin{tabular}{|c|c|c|c|c|c|}
\hline \multirow[b]{3}{*}{ Tissue or ratio } & \multicolumn{5}{|c|}{ Biodistribution of ${ }^{131} \mathrm{I}-\mathrm{FIAU}$ (mean $\pm \mathrm{SD} \% \mathrm{ID} / \mathrm{g}$ ) } \\
\hline & \multicolumn{4}{|c|}{ After cell transplantation via: } & \multirow[b]{2}{*}{ In normal rats } \\
\hline & Local injection & Ventricular injection & Carotid artery injection & Tail vein injection & \\
\hline Infarcted brain & $0.124 \pm 0.013$ & $0.052 \pm 0.004$ & $0.061 \pm 0.002$ & $0.059 \pm 0.005$ & $0.005 \pm 0.001$ \\
\hline Contralateral brain & $0.025 \pm 0.004$ & $0.021 \pm 0.001$ & $0.031 \pm 0.005$ & $0.029 \pm 0.005$ & $0.004 \pm 0.001$ \\
\hline Thyroid & $0.724 \pm 0.030$ & $0.640 \pm 0.042$ & $0.671 \pm 0.047$ & $0.654 \pm 0.116$ & $0.614 \pm 0.065$ \\
\hline Lung & $0.043 \pm 0.012$ & $0.025 \pm 0.006$ & $0.045 \pm 0.013$ & $0.050 \pm 0.005$ & $0.035 \pm 0.004$ \\
\hline Heart & $0.045 \pm 0.003$ & $0.047 \pm 0.003$ & $0.062 \pm 0.004$ & $0.045 \pm 0.008$ & $0.024 \pm 0.011$ \\
\hline Liver & $0.061 \pm 0.015$ & $0.052 \pm 0.001$ & $0.045 \pm 0.005$ & $0.043 \pm 0.005$ & $0.035 \pm 0.004$ \\
\hline Stomach & $0.085 \pm 0.016$ & $0.073 \pm 0.010$ & $0.080 \pm 0.010$ & $0.062 \pm 0.009$ & $0.443 \pm 0.269$ \\
\hline Pancreas & $0.047 \pm 0.012$ & $0.045 \pm 0.007$ & $0.024 \pm 0.003$ & $0.050 \pm 0.017$ & $0.034 \pm 0.006$ \\
\hline Spleen & $0.070 \pm 0.024$ & $0.078 \pm 0.009$ & $0.092 \pm 0.021$ & $0.085 \pm 0.035$ & $0.094 \pm 0.011$ \\
\hline Kidney & $0.083 \pm 0.044$ & $0.114 \pm 0.015$ & $0.121 \pm 0.014$ & $0.107 \pm 0.007$ & $0.112 \pm 0.012$ \\
\hline Muscle & $0.044 \pm 0.008$ & $0.062 \pm 0.013$ & $0.045 \pm 0.006$ & $0.045 \pm 0.008$ & $0.049 \pm 0.008$ \\
\hline Bone & $0.034 \pm 0.006$ & $0.051 \pm 0.003$ & $0.033 \pm 0.003$ & $0.041 \pm 0.002$ & $0.045 \pm 0.011$ \\
\hline Intestine & $0.110 \pm 0.020$ & $0.094 \pm 0.017$ & $0.114 \pm 0.019$ & $0.100 \pm 0.029$ & $0.113 \pm 0.025$ \\
\hline Blood & $0.017 \pm 0.004$ & $0.012 \pm 0.005$ & $0.015 \pm 0.004$ & $0.029 \pm 0.031$ & $0.015 \pm 0.005$ \\
\hline Ratio for infarcted brain to blood & $7.765 \pm 3.081$ & $3.429 \pm 1.344$ & $3.779 \pm 1.042$ & $3.328 \pm 1.602$ & $0.351 \pm 0.059$ \\
\hline
\end{tabular}

time, but the ratio of counts in bilateral brain tissues peaked (6.63) $24 \mathrm{~h}$ after ${ }^{131}$ I-FIAU injection.

\section{SPECT Imaging}

With whole-body imaging (Fig. 5) of experimental MCAO rat models, the liver and bladder were clearly imaged. The level of radioactivity accumulation in the brain was low but increased gradually over time.

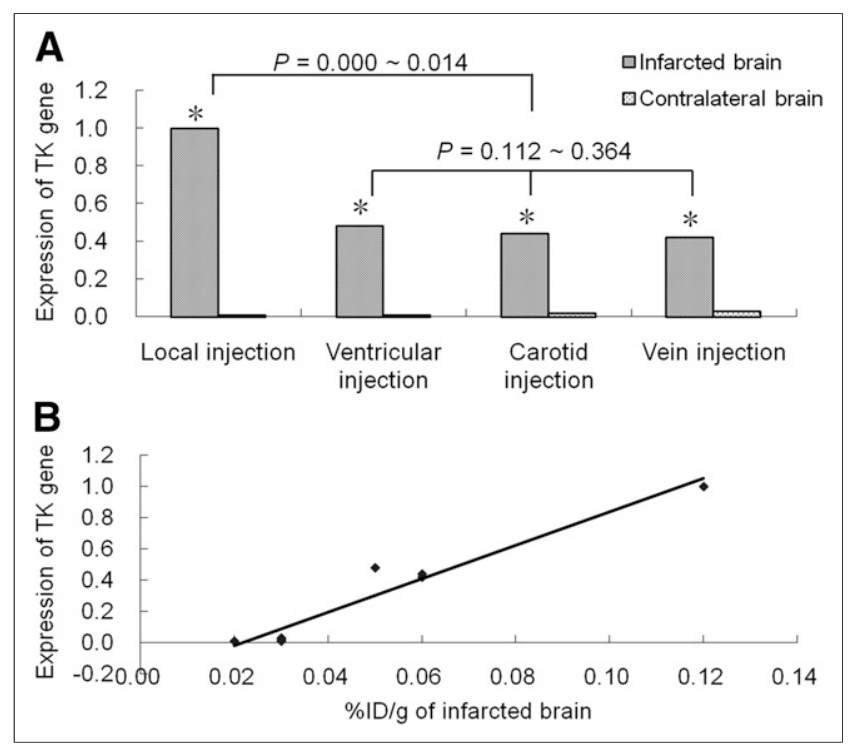

FIGURE 2. TK gene expression in different cell injection groups at gene level. (A) Level of expression of TK gene in infarcted brain tissue was higher than that in contralateral brain tissue in all groups $\left({ }^{*} P<0.001\right)$, and highest level of expression of TK gene was seen in local injection group. (B) TK gene expression from real-time quantitative polymerase chain reaction showed positive correlation with $\% \mathrm{ID} / \mathrm{g}$ in brain tissue $\left(R^{2}=0.971 ; P<0.001\right)$.

\section{DISCUSSION}

In the present study, we showed that the local injection of BMSCs into the brain rather than injection into other sites led to greater radioactivity accumulation in infarcted brain tissue, indicating an increase in the level of BMSCs surrounding the infarcted tissue. Therefore, the present

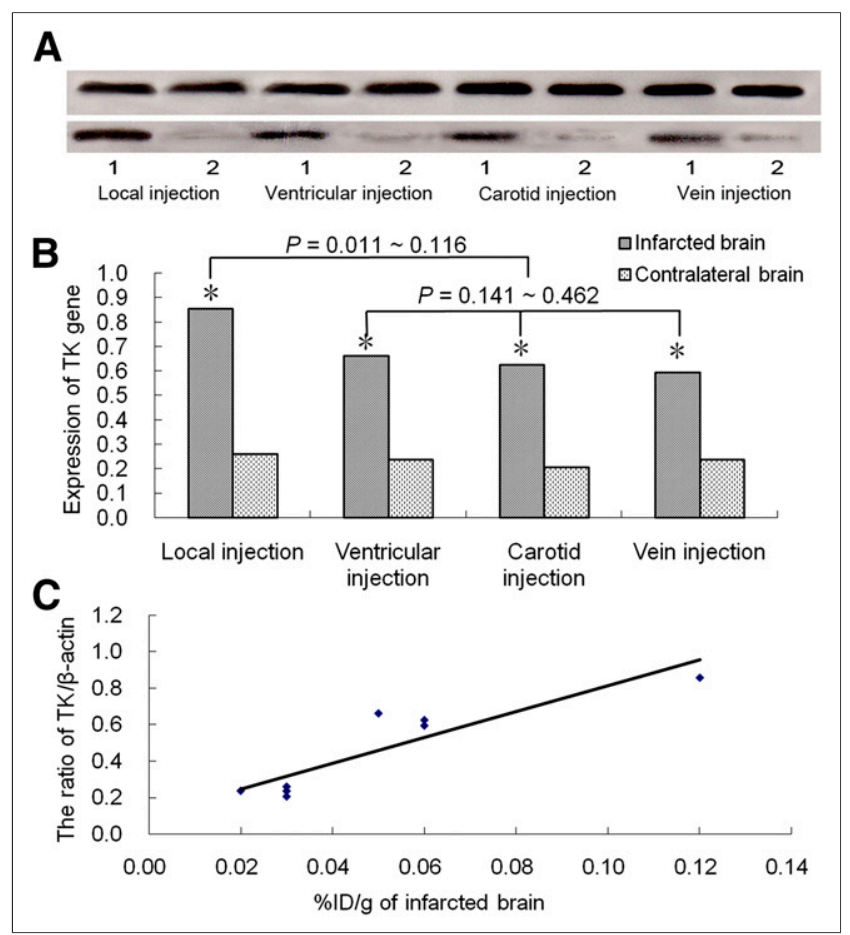

FIGURE 3. TK gene expression in different cell injection groups at protein level. (A) Western blot images of TK gene expression. (B) Ratio of TK to $\beta$-actin in infarcted brain tissue was higher than that in contralateral brain tissue for all groups $\left({ }^{*} P<0.001\right)$, and highest ratio was seen in local injection group. (C) Ratio of TK to $\beta$-actin on Western blotting and \% ID/g correlated well $\left(R^{2}=0.899 ; P=0.002\right)$. 


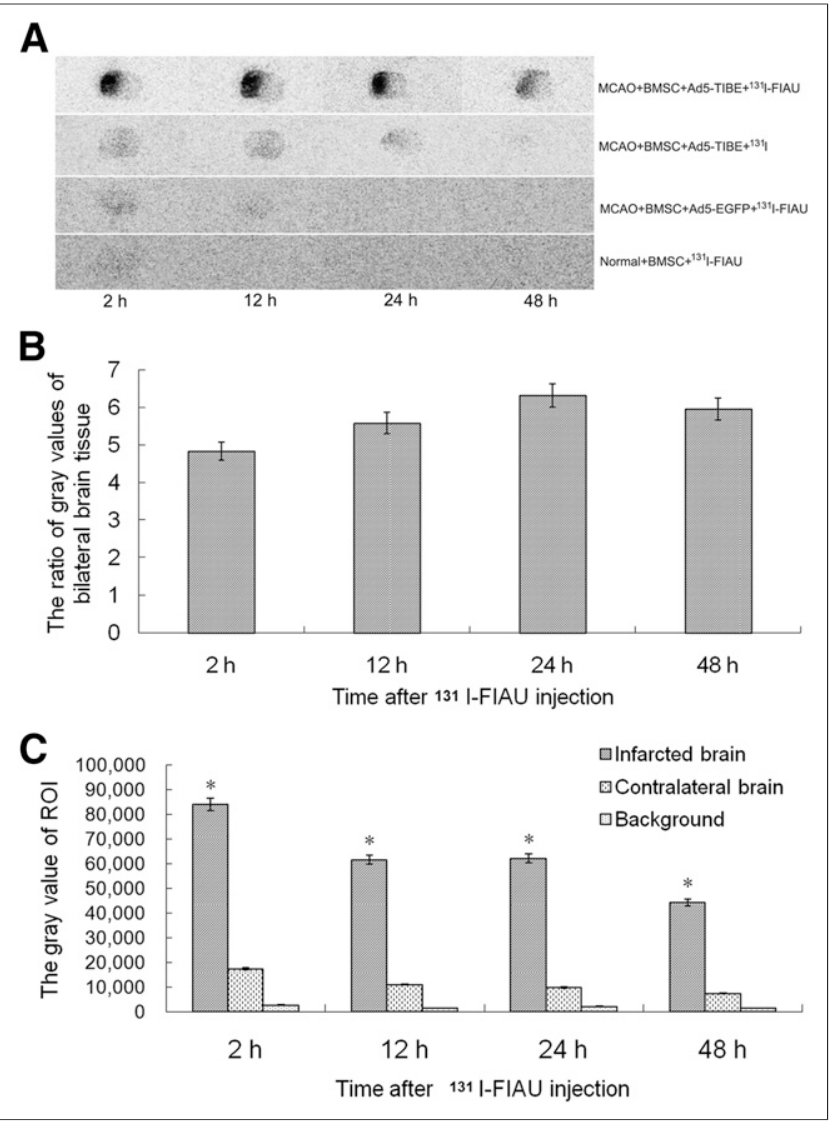

FIGURE 4. (A) ARG images of brain tissue showed obvious radioactivity accumulation in infarcted brain of group 1 (top row) relative to other groups, and counts decreased over time. (B) Ratio of counts in bilateral brain tissues reached its peak (6.63) at $24 \mathrm{~h}$. (C) Counts in infarcted brain in group 1 were higher than those in contralateral brain and background $\left({ }^{*} P<0.001\right)$. Gray value $=$ counts of region of interest on ARG images; $\mathrm{ROI}=$ region of interest.

study suggests that local injection into the brain is an optimal cell transplantation path.

An increasing amount of evidence has shown that stem cell therapy can reduce ischemic brain damage and improve brain function, but the mechanism remains unclear and a debate still exists regarding the optimal method of cell delivery. Therefore, with the goal of comparing different means of transplantation, we used 4 methods of cell transplantation concurrently in the present study. Some differences among the various methods of cell transplantation were seen. The $\% \mathrm{ID} / \mathrm{g}$ in infarcted brain tissue in rats receiving the injection into the brain was $0.124 \pm 0.013$; this value was significantly higher than those in rats receiving the injection into the lateral ventricle $(0.052 \pm 0.004)$, carotid artery $(0.061 \pm 0.002)$, and tail vein $(0.059 \pm 0.005)$. The reason for this difference may be related to the amount of surviving cells in the target brain tissue. Some related studies verified this hypothesis. The percentages of cells surviving in ischemia boundary zones were reported to reach approximately $14 \%$ $\left[\left(5 \times 10^{4}-5.9 \times 10^{4}\right) / 4 \times 10^{5}\right], 21 \%\left(433,700 / 2 \times 10^{6}\right)$, and $0.7 \%\left(7,500 / 1 \times 10^{6}\right)-1.1 \%\left(31,600 / 3 \times 10^{6}\right)$ when injected via intracerebral (3), intracarotid (4), and tail vein (18) routes, respectively.
Moreover, in our study, little difference was seen among the groups that received injection via blood. This finding may be related to the short observation time in the present study. In other studies, all of the rats were injected with ${ }^{131}$ I-FIAU for biodistribution experiments, and the cell numbers were counted $14 \mathrm{~d}$ after cell injection $(3,4,18)$. Twenty-four hours may be not sufficient for cells to migrate to the target brain tissue. The fate of the cells may be different. Some studies suggested that exposure to the brain microenvironment may cause BMSCs grafted into brain tissue to differentiate into microglia, macroglia, and astrocytes in normal and ischemic brains of adult animals (20-23). Intravenously transplanted BMSCs have been shown to be easily trapped by the liver, spleen, and lung in the delivery process $(24,25)$, and BMSCs transplanted via the intracarotid route are at risk for local incarceration (4). The different fates of transplanted cells in the delivery process is another reason for the differences among the injection methods. Intraparenchymal injection can also provide precision with regard to graft placement (9). Therefore, we suggest that local injection into the brain is the optimal means of cell delivery. Our results also demonstrate that there was no significant difference in the \% ID/g in bilateral brain tissues whether BMSCs were injected into the tail vein or into the carotid artery; this finding may be related to cell volume, cell density, and artery embolism.

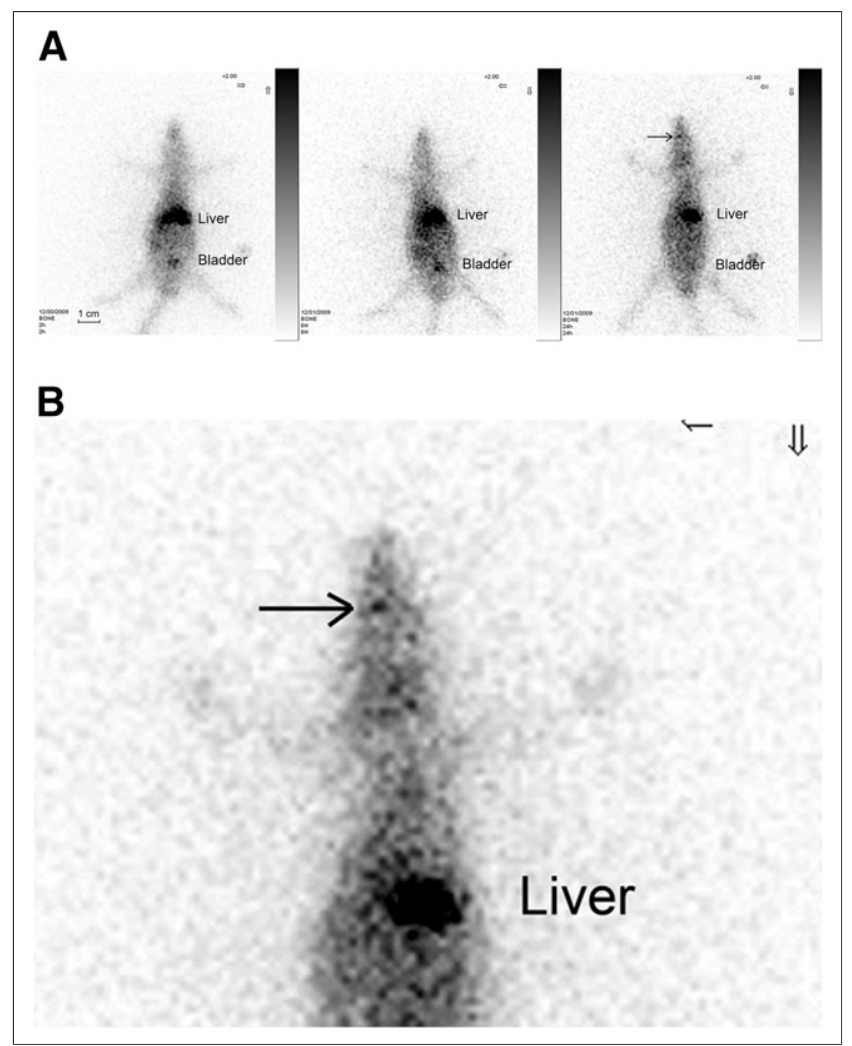

FIGURE 5. SPECT images of MCAO models with transplanted BMSCs. (A) Liver and bladder were clearly imaged. Level of accumulation in brain (arrow) was low but increased gradually over time. (B) Local enlargement of A. Greater radioactivity accumulation was seen at cell injection site (arrow). 
Previous studies showed a positive correlation between reporter gene (HSV1-tk) and therapeutic gene (BDNF) expression at the cellular level (26), indicating the coexpression of TK and BDNF. In the present study, HSV1-tk messenger RNA and protein expression also showed a positive correlation with \% ID/g in MCAO models; this result, in combination with the results of the biodistribution experiments, may have been attributable to the specific phosphorylation of ${ }^{131} \mathrm{I}-$ FIAU by the product of the expression of the HSV1-tk gene.

Radioactivity accumulation on ARG images occurred only in experimental rat models, not in controls, indicating that ${ }^{131}$ I-FIAU was not taken up by the normal brain and the BMSCs; the infarcted brain and the product of the expression of the HSV1-tk gene did not take up ${ }^{131} \mathrm{I}$. The target-to-nontarget ratio reached a peak (6.63) $24 \mathrm{~h}$ after ${ }^{131}$ I-FIAU was injected. Meanwhile, the radioactivity in the contralateral brain was close to the background. Therefore, $24 \mathrm{~h}$ after injection of ${ }^{131}$ I-FIAU may be the optimal time for imaging in vivo.

In the present study, radioactivity in the infarcted brain tissue (relative to the controls) and the accumulation of radioactivity in the brain were largely attributed to the specific phosphorylation of ${ }^{131}$ I-FIAU by the product of the expression of the HSV1-tk gene; the findings showed indirectly that experimental cerebral infarction led to the destruction of the blood-brain barrier, providing the necessary condition for radionuclide imaging of the reporter gene. Despite the limited resolution of clinical SPECT, mild radioactivity accumulation in brain tissue was still demonstrated. Future studies could address the deficiencies of SPECT imaging by performing tomography and local amplification.

Additionally, in the present study, a recombinant adenovirus was used as the vector for carrying a reporter gene and a therapeutic gene. It has a large capacity, can be inserted as a 7.5-kilobase fragment of an exogenous gene (27), and easily achieves a high yield. This vector can meet the needs of follow-up experiments. In future studies, we intend to apply lentivirus as a carrier because it may integrate into DNA, making in vivo monitoring for a longer time possible.

Additional studies to monitor transplanted cells with SPECT or PET/CT in vivo and to determine improvements in brain function through behavioral testing may delineate the therapeutic benefits of cerebral blood flow imaging and brain functional imaging.

\section{CONCLUSION}

The present study confirmed the feasibility of HSV1-tk${ }^{131}$ I-FIAU as a reporter gene-probe system for monitoring the fate of BMSCs transplanted into experimental MCAO rat models with SPECT/CT or PET/CT imaging. Local injection of stem cells may provide an optimal means for cell transplantation, and imaging with ${ }^{131}$ I-FIAU $24 \mathrm{~h}$ after injection provides peak target-to-nontarget count ratios.

\section{DISCLOSURE}

The costs of publication of this article were defrayed in part by the payment of page charges. Therefore, and solely to indicate this fact, this article is hereby marked "advertisement" in accordance with 18 USC section 1734. This study was supported by the National Natural Science Foundation of China (No. 30770605) and Fundamental Research Funds for the Central Universities (No. 2011JC011). No other potential conflict of interest relevant to this article was reported.

\section{REFERENCES}

1. Li Y, Chen J, Chen XG, et al. Human marrow stromal cell therapy for stroke in rat: neurotrophins and functional recovery. Neurology. 2002;59:514-523.

2. Zhao LR, Duan WM, Reyes M, et al. Human bone marrow stem cells exhibit neural phenotypes and ameliorate neurological deficits after grafting into the ischemic brain of rats. Exp Neurol. 2002;174:11-20.

3. Chen J, Li Y, Wang L, et al. Therapeutic benefit of intracerebral transplantation of bone marrow stromal cells after cerebral ischemia in rats. J Neurol Sci. 2001;189:49-57.

4. Li Y, Chen J, Wang L, et al. Treatment of stroke in rat with intracarotid administration of arrow stromal cells. Neurology. 2001;56:1666-1672.

5. Zheng W, Honmou O, Miyata K, et al. Therapeutic benefits of human mesenchymal stem cells derived from bone marrow after global cerebral ischemia. Brain Res. 2010;1310:8-16.

6. Kurozumi K, Nakamura K, Tamiya T, et al. BDNF gene-modified mesenchymal stem cells promote functional recovery and reduce infarct size in the rat middle cerebral artery occlusion model. Mol Ther. 2004;9:189-197.

7. Ikeda N, Nonoguchi N, Zhao MZ, et al. Bone marrow stromal cells that enhanced fibroblast growth factor-2 secretion by herpes simplex virus vector improve neurological outcome after transient focal cerebral ischemia in rats. Stroke. 2005;36:2725-2730.

8. Adonai N, Nguyen KN, Walsh J, et al. Ex vivo cell labeling with ${ }^{64} \mathrm{Cu}$-pyruvaldehyde-bis $\left(N_{4}\right.$-methylthiosemicarbazone) for imaging cell trafficking in mice with positron-emission tomography. Proc Natl Acad Sci USA. 2002;99:3030-3035.

9. Tran-Dinh A, Kubis N, Tomita Y, et al. In vivo imaging with cellular resolution of bone marrow cells transplanted into the ischemic brain of a mouse. Neuroimage. 2006;31:958-967.

10. Tang Y, Shah K, Messerli SM, et al. In vivo tracking of neural progenitor cell migration to glioblastomas. Hum Gene Ther. 2003;14:1247-1254.

11. Walczak P, Zhang J, Gilad AA, et al. Dual-modality monitoring of targeted intraarterial delivery of mesenchymal stem cells after transient ischemia. Stroke. 2008;39:1569-1574.

12. Tjuvajev JG, Stockhammer G, Desai R, et al. Imaging the expression of transfected genes in vivo. Cancer Res. 1995;55:6126-6132.

13. Gambhir SS, Barrio JR, Wu L, et al. Imaging of adenoviral-directed herpes simplex virus type 1 thymidine kinase reporter gene expression in mice with radiolabeled ganciclovir. J Nucl Med. 1998;39:2003-2011.

14. Jacobs A, Bräunlich I, Graf R, et al. Quantitative kinetics of $\left[{ }^{124} I\right]$ FIAU in cat and man. J Nucl Med. 2001;42:467-475.

15. Kuge Y, Minematsu K, Yamaguchi T, et al. Nylon monofilament for intraluminal middle cerebral artery occlusion in rats. Stroke. 1995;26:1655-1657.

16. Longa EZ, Weinstein PR, Carlson S, et al. Reversible middle cerebral artery occlusion without craniectomy in rats. Stroke. 1989;20:84-91.

17. Honma $\mathrm{T}$, Honmou $\mathrm{O}$, Iihoshi $\mathrm{S}$, et al. Intravenous infusion of immortalized human mesenchymal stem cells protects against injury in a cerebral ischemia model in adult rat. Exp Neurol. 2006;199:56-66.

18. Chen J, Li Y, Wang L, et al. Therapeutic benefit of intravenous administration of bone marrow stromal cells after cerebral ischemia in rats. Stroke. 2001;32:1005-1011.

19. Lan X, Liu Y, He Y, et al. Autoradiography study and SPECT imaging of reporter gene HSV1-tk expression in heart. Nucl Med Biol. 2010;37:371-380.

20. Chen J, Li Y, Chopp M. Intracerebral transplantation of bone marrow with BDNF after MCAo in rat. Neuropharmacology. 2000;39:711-716.

21. Eglitis MA, Mezey E. Hematopoietic cells differentiate into both microglia and macroglia in the brains of adult mice. Proc Natl Acad Sci USA. 1997;94:4080-4085.

22. Kopen GC, Prockop DJ, Phinney DG. Marrow stromal cells migrate throughout forebrain and cerebellum, and they differentiate into astrocytes after injection into neonatal mouse brains. Proc Natl Acad Sci USA. 1999;96:10711-10716.

23. Azizi SA, Stokes D, Augelli BJ, et al. Engraftment and migration of human bone marrow stromal cells implanted in the brains of albino rats: similarities to astrocyte grafts. Proc Natl Acad Sci USA. 1998;95:3908-3913.

24. Detante O, Moisan A, Dimastromatteo J, et al. Intravenous administration of ${ }^{99 m}$ Tc-HMPAO-labeled human mesenchymal stem cells after stroke: in vivo imaging and biodistribution. Cell Transplant. 2009;18:1369-1379.

25. Hauger O, Frost EE, van Heeswijk R, et al. MR evaluation of the glomerular homing of magnetically labeled mesenchymal stem cells in a rat model of nephropathy. Radiology. 2006;238:200-210.

26. Zhang BQ, Wu T, Sun X, et al. Uptake of ${ }^{131}$ I-FIAU in BMSCs infected by adenovirus vector-mediated HSV1-TK. Nucl Technol. 2010;33:303-307.

27. Mitani K, Kubo S. Adenovirus as an integrating vector. Curr Gene Ther. 2002;2:135-144. 